\title{
The Wigner-Ville Distribution Based on the Offset Linear Canonical Transform Domain
}

\author{
Didar Urynbassarova $^{1}$, Altyn Urynbassarova ${ }^{2}$ and Ebrahim Al-Hussam ${ }^{3, *}$ \\ ${ }^{1}$ School of Mathematics and Statistics, Beijing Institute of Technology, Beijing, China \\ ${ }^{2}$ School of Finance and Technology, Narxoz University, Almaty, Kazakhstan \\ ${ }^{3}$ Fission Technology Co., Beijing, China \\ ${ }^{*}$ Corresponding author
}

\begin{abstract}
The Wigner-Ville distribution (WVD) in the offset linear canonical transform (OLCT) domain (WOL) is a tool for signal processing and optics, which has the advantages of the OLCT and good properties of WVD. In this paper, a more simple definition of the WOL is introduced, without changing the instantaneous autocorrelation function to generalization form. Moreover, some new and important properties of the WOL are derived.
\end{abstract}

Keywords-Wigner-Ville distribution; offset linear canonical transform; linear canonical transform; translation; modulation; instantaneous frequency; uncertainty principle

\section{INTRODUCTION}

The offset linear canonical transform (OLCT) [1]-[4] is a linear integral transform with six parameters $\left(a, b, c, d, u_{0}, \omega_{0}\right)$, which is also known under different names, the inhomogeneous canonical transform [1] or the special affine transformation [5]. It has found wide applications in several areas, including applied mathematics, optics, engineering, and signal processing. Many properties of the OLCT have been intensively researched and studied. Widely used operations, such as the Fourier transform (FT) [6], fractional Fourier transform (FrFT) [7], linear canonical transform (LCT) [4], [8]-[16], Fresnel transform [17], Laplace transform, and time scaling operations are all special cases of the OLCT.

The Wigner-Ville distribution (WVD) is a special type of quasi-probability distribution, which was first proposed in physics by Wigner. Later Ville re-derived it as a quadratic representation of the local time-frequency energy of a signal. Among well-known time-frequency methods, such as the chirp-Fourier transform [18], the Radon-ambiguity transform [19], and the Wigner-Hough transform [20], the WVD can furnish higher clarity in the linear-frequency-modulated (LFM) signal detection and parameter estimation, which is important for the signal processing and engineering community.

Based on the properties of the LCT and the WVD, Pei and Ding [9] investigated WVD of the signal $F_{a, b, c, d}(u)$. Unlike the definition of WVD associated with the LCT in [9], Bai et al. in [10] proposed generalized kind of WVD in the LCT domain (WDL), which can be thought as the affine transform of the autocorrelation function of $f(x)$ in the time-frequency plane. In [4] and [14], authors have defined a kind of novel WVD in the LCT domain (WL) separately. The WL is a new signal processing tool, which has the elegance and simplicity in marginal property and affine transformation relationships associated with the LCT comparable to the WVD. Also in [4] proposed further generalization of the WL- the WVD in the OLCT domain (WOL), by using generalized form of the instantaneous autocorrelation function. But authors have not discussed the WOL in depth enough. This paper presents a more simplified definition of the WOL by using the classical instantaneous autocorrelation function instead of using generalized form of the instantaneous autocorrelation function shown in [4]. Then, we deduce some new properties of the WOL with its detailed proves. Furthermore, we provide a new way to calculate the instantaneous frequency.

This paper is organized to include at first the introduction, followed by "Preliminary" section which briefly introduce the review of the related theory of the OLCT, the WVD, and the previous research outputs. In Section III "WVD in the OLCT domain", a novel definition of WVD associated with the OLCT is proposed. The new properties of WOL are investigated in details in Section IV "Properties of the WOL". Section V concludes this paper.

\section{PRELIMINARY}

The preliminary spots light on reviewing the already established definitions and previous researches.

\section{A. The Offset Linear Canonical Transform (OLCT)}

The OLCT [1]-[4] is a six-parameter class of linear integral transform. It is more flexible and general than the LCT for its two extra parameters $u_{0}$ and $\omega_{0}$ which correspond to time shifting and frequency modulation, respectively.

The OLCT with real parameters of $A=\left(a, b, c, d, u_{0}, \omega_{0}\right)$ of a signal $f(t)$ is defined by [1]-[4]

$$
\begin{aligned}
& F_{A}(u)=O_{A}[f(t)](u) \\
& = \begin{cases}\int_{\mathfrak{R}} f(t) \sqrt{\frac{1}{i 2 \pi b}} e^{\frac{i}{2 b} d u_{0}^{2}} e^{\frac{i}{2 b}\left[a t^{2}+2 t\left(u_{0}-u\right)-2 u\left(d u_{0}-b \omega_{0}\right)+d u^{2}\right]} d t, b \neq 0 \\
\sqrt{d} e^{\frac{i}{2} c d\left(u-u_{0}\right)^{2}+i \omega_{0} u} f\left[d\left(u-u_{0}\right)\right], & b=0,\end{cases}
\end{aligned}
$$


and $a d-b c=1$. The definition for case $b=0$ is the limit of the integral in above for the case $b \neq 0$ as $|b| \rightarrow 0$. Therefore, from now we shall confine our attention to OLCT for $b \neq 0$. And without loss generality, we always assume $b>0$ in the following sections of the paper.

The OLCT as a generalization of many other linear transforms has had a great development and has found wide applications in applied mathematics, signal processing and optics. The OLCT can solve some problems that cannot be solved well by its special cases. In this point, the development of the relevant theorems for OLCT helps to achieve more insights and to serve knowledge exchange from one subject to others.

For more definitions and properties of the OLCT, interested readers can refer to [3].

\section{B. The Wigner-Ville Distribution (WVD)}

The classical WVD of a signal $f(t)$ defined as the FT of $R_{f}(t, \tau)$ for $\tau$ [4], [8], [10]-[14], [16]

$$
W(t, \omega)=\int_{\Re} R_{f}(t, \tau) e^{-i \omega \tau} d \tau,
$$

where $R_{f}(t, \tau)$ is the instantaneous autocorrelation function of a signal $f(t)$

$$
R_{f}(t, \tau)=f\left(t+\frac{\tau}{2}\right) f^{*}\left(t-\frac{\tau}{2}\right)
$$

The WVD is one of the most useful time-frequency analysis tool and has a series of good properties. Some properties of WVD are listed in [8], [10], [11].

\section{Previous Research Outputs}

With the development of the modern signal processing technology, a series of novel time-frequency representations have been proposed through combining the classical signal processing tools with the LCT [4], [9]-[14].

The WVD associated with the LCT (LCWD) given in [9] is a tool for the separation of multi-component signals. It is defined as

$$
W D_{A}(u, v)=\int_{\Re} F_{A}\left(u+\frac{\tau}{2}\right) F_{A}^{*}\left(u-\frac{\tau}{2}\right) e^{-i v \tau} d \tau,
$$

where $F_{A}(u)$ is the LCT of signal $f(t)$ with parameter matrix $A$. The LCT is equivalent to an affine or rotation operation for the WVD

$$
W D_{A}(u, v)=W D_{f}(d u-b v,-c u+a v)
$$

where $W D_{f}$ is the WVD of $f(t)$ [9].
According to (2), we know that the WVD is the FT of instantaneous autocorrelation function. Recently, Bai et. al obtained WVD in the LCT domain (WDL) by substituting the orthogonal kernel of the FT with the non-orthogonal kernel of the LCT [10]

$$
W_{A=(a, b, c, d)}^{f}(t, u)=\int_{\Re} R_{f}(t, \tau) \sqrt{\frac{1}{i 2 \pi b}} e^{\frac{i}{2}\left(\frac{a}{b} \tau^{2}-\frac{2}{b} u \tau+\frac{d}{b} u^{2}\right)} d \tau .
$$

Although, in [10]-[12], [16] authors investigated the various properties and applications of the WDL. The WDL is a generalized form of LCWD and WVD.

In order to estimate QFM signal parameters, Song et al. defined a new kind of WVD - the generalized WVD based on the LCT (GWDL) [12]. Zhang proposed the WVD based on the generalized convolution in the LCT domain (LWD) [13]. However, the WDL, GWDL, and LWD have a bit of complexity comparing to the WVD and have no affine transformation relationships between the LCT and WDL, GWDL, and LWD. Focusing on this issues, Urynbassarova et al. and Zhang have proposed a kind of novel WVD based on LCT domain (WL) separately in [4], [14]. They shows the WL is a simpler time-frequency analysis tool than the LWD, WDL and LCWD. Also in [4] proposed further generalization of the WL-the WVD based on the OLCT domain (WOL) by using generalized form of the instantaneous autocorrelation function. The WOL is a new signal processing tool, which has the elegance and simplicity in marginal property and affine relation associated with the OLCT in comparison with the WVD. But authors have not discussed the WOL in depth enough. This paper serves to simplify more the definition of the WOL and deduce new properties of WOL with its proves.

\section{The NEW DEFINITION OF WVD IN THE OLCT DOMAIN}

In this Section, the cross and auto WOL are defined and some essential properties are deduced.

Definition. The cross WOL of signals $f$ and $g$ for the parameter matrix $A=\left(a, b, c, d, u_{0}, \omega_{0}\right)$ is defined as follows

$$
W O L_{f, g}(t, u)=\int_{\Re} f\left(t+\frac{\tau}{2}\right) g^{*}\left(t-\frac{\tau}{2}\right) e^{\frac{i a}{b} \tau t} e^{\frac{i}{b} \tau\left(u_{0}-u\right)} d \tau
$$

If $f=g$ then $W_{f, f}(t, u)=W O L_{f}(t, u)$ is called the auto WOL. That is,

$$
W O L_{f, f}(t, u)=\int_{\mathfrak{R}} f\left(t+\frac{\tau}{2}\right) f^{*}\left(t-\frac{\tau}{2}\right) e^{\frac{i a}{b} \tau t} e^{\frac{i}{b} \tau\left(u_{0}-u\right)} d \tau
$$

Both the cross WOL and auto WOL are referred to be as simple as the WOL and are simplified as the WOL of signal $f(t)$ and denoted by $\operatorname{WOL}_{f}(t, u)$.

The relationship between WL and WOL is easy to be verified; when the parameter $A$ is reduced to 
$A=(a, b, c, d, 0,0)$, the WOL is reduced to the $\mathrm{WL}$

$$
W O L_{f}^{(a, b, c, d, 0,0)}(t, u)=W L_{f}(t, u) .
$$

In this sense, the WOL is the generalization of the WL.

Obviously, when the parameter matrix has the special form $A=(0,1,-1,0,0,0)$, the WOL is reduced to the WVD

$$
W O L_{f}^{(0,1,-1,0,0,0)}(t, u)=W(t, u) .
$$

From (4) and (5) we can see that the WOL is a generalization of the WL and WVD.

\section{THE Properties OF THE WOL}

In this Section, the new properties of the WOL, such as translation, modulation, multiplied signal, and instantaneous frequency are investigated. Besides, the section illustrates the uncertainty principle of the WOL.

\section{A. The Properties of the WOL}

1) Translation property: The translation property of WOL for $f^{\prime}(t)=f(t-\lambda)$ has the form

$$
W O L_{f^{\prime}}(t, u)=W O L_{f}(t-\lambda, u-a \lambda) .
$$

Proof. From definition of the WOL (3) we have

$$
\begin{aligned}
W O L_{f^{\prime}}(t, u) & =\int_{\Re} f\left(t+\frac{\tau}{2}-\lambda\right) f^{*}\left(t-\frac{\tau}{2}-\lambda\right) e^{\frac{i a}{b} t t} e^{-\frac{i}{b}\left(u-u_{0}\right) \tau} d \tau \\
& =\int_{\Re} f\left(t+\frac{\tau}{2}-\lambda\right) f^{*}\left(t-\frac{\tau}{2}-\lambda\right) \\
& \cdot e^{\frac{i a}{b} t t} e^{-\frac{i}{b}\left(u-u_{0}\right) \tau} e^{-\frac{i a}{b} \tau \lambda} e^{\frac{i a}{b} \tau \lambda} d \tau \\
= & \int_{\Re} f\left((t-\lambda)+\frac{\tau}{2}\right) f^{*}\left((t-\lambda)-\frac{\tau}{2}\right) \\
& \cdot e^{\frac{i a}{b} \tau(t-\lambda)} e^{-\frac{i}{b}\left(\left(u-u_{0}\right)-a \lambda\right) \tau} d \tau \\
& =W_{f}(t-\lambda, u-a \lambda) .
\end{aligned}
$$

Proof of this property is completed.

2) Modulation property: For $f^{\prime}(t)=f(t) e^{2 \pi i u_{1} t}$, then

$$
\begin{aligned}
W O L_{f^{\prime}}(t, u) & =\int_{\mathfrak{R}} f\left(t+\frac{\tau}{2}\right) e^{2 \pi i u_{1}\left(t+\frac{\tau}{2}\right)} f^{*}\left(t-\frac{\tau}{2}\right) e^{-2 \pi i u_{1}\left(t-\frac{\tau}{2}\right)} \\
& \cdot e^{\frac{i a}{b} \tau t} e^{-\frac{i}{b}\left(u-u_{0}\right) \tau} d \tau \\
& =\int_{\mathfrak{R}} f\left(t+\frac{\tau}{2}\right) f^{*}\left(t-\frac{\tau}{2}\right) e^{2 \pi i u_{1} \tau} e^{\frac{i a}{b} t t} e^{-\frac{i}{b}\left(u-u_{0}\right) \tau} d \tau \\
& =\operatorname{WOL}_{f}\left(t, u-2 \pi u_{1} b\right) .
\end{aligned}
$$

3) Multiplied signal: For $f(t)=g(t) h(t)$,

$$
\begin{aligned}
W O L_{f}(t, u)= & \int_{\mathfrak{R}} g\left(t+\frac{\tau}{2}\right) h\left(t+\frac{\tau}{2}\right) g^{*}\left(t-\frac{\tau}{2}\right) h^{*}\left(t-\frac{\tau}{2}\right) \\
& \cdot e^{\frac{i a}{b} t t} e^{-\frac{i}{b}\left(u-u_{0}\right) \tau} d \tau \\
= & \int_{\mathfrak{R}^{2}} g\left(t+\frac{\tau}{2}\right) g^{*}\left(t-\frac{\tau}{2}\right) h\left(t+\frac{\tau^{\prime}}{2}\right) h^{*}\left(t-\frac{\tau^{\prime}}{2}\right) \\
& \cdot e^{\frac{i a}{b} \pi t} e^{-\frac{i}{b}\left(u-u_{0}\right) \tau} \delta\left(\tau^{\prime}-\tau\right) d \tau d \tau^{\prime} \\
= & \frac{1}{2 \pi|b|} \int_{\mathfrak{R}^{3}} g\left(t+\frac{\tau}{2}\right) g^{*}\left(t-\frac{\tau}{2}\right) h\left(t+\frac{\tau^{\prime}}{2}\right) h^{*}\left(t-\frac{\tau^{\prime}}{2}\right) \\
& \cdot e^{\frac{i a}{b} t t} e^{-\frac{i}{b}\left(u-u_{0}\right) \tau} e^{\frac{i u^{\prime} \tau}{b}-\frac{i u^{\prime} \tau^{\prime}}{b}} d u^{\prime} d \tau d \tau^{\prime} \\
= & \frac{1}{2 \pi|b|} \int_{\mathfrak{R}} W L_{g}\left(t, u-u^{\prime}\right) W_{h}\left(t, 2 \pi b u^{\prime}\right) d u^{\prime} .
\end{aligned}
$$

4) Instantaneous frequency: For any signal $f(t)=|f(t)| e^{i \varphi(t)}$

$$
u_{i}(t)=\int_{\mathfrak{R}} u W O L_{f}(t, u) d u / \int_{\mathfrak{R}} W O L_{f}(t, u) d u .
$$

Here, the denominator of above equation is time marginal property [4], so the $u_{i}(t)$ can be rewritten as follows

$$
\begin{aligned}
& \frac{\frac{1}{2 \pi|b|} \int_{\mathfrak{R}^{2}} u \cdot f\left(t+\frac{\tau}{2}\right) f^{*}\left(t-\frac{\tau}{2}\right) e^{i\left[\frac{a}{b} \pi t-\frac{\left(u-u_{0}\right)}{b} \tau\right]} d \tau d u}{|f(t)|^{2}} \\
& =\frac{\frac{1}{2 \pi|b|} \frac{b}{i} \int_{\mathfrak{R}^{2}} \frac{\partial\left(f\left(t+\frac{\tau}{2}\right) f^{*}\left(t-\frac{\tau}{2}\right) e^{\frac{i a}{b} t t} e^{\frac{i}{b} u_{0} \tau}\right)}{\partial \tau} e^{-\frac{i}{b} u \tau} d \tau d u}{|f(t)|^{2}} \\
& =\frac{\frac{b}{i} \int_{\Re} \frac{\partial\left(f\left(t+\frac{\tau}{2}\right) f^{*}\left(t-\frac{\tau}{2}\right) e^{\frac{i a}{b} t} e^{\frac{i}{b} u_{0} \tau}\right)}{\partial \tau} \delta(\tau) d \tau}{|f(t)|^{2}}
\end{aligned}
$$




$$
\begin{aligned}
& =\left.\frac{b}{i|f(t)|^{2}} \frac{\partial\left(f\left(t+\frac{\tau}{2}\right) f^{*}\left(t-\frac{\tau}{2}\right) e^{\frac{i a}{b} \tau t} e^{\frac{i}{b} u_{0} \tau}\right)}{\partial \tau}\right|_{\tau=0} \\
& =\frac{b}{i}\left(\varphi^{\prime}(t)+\frac{a t}{b}\right) .
\end{aligned}
$$

\section{B. The Uncertainty Principle of the $W O L$}

The uncertainty principle [8], [15], [16] plays an important role in physics and communication. It is helpful for analyzing the characteristic of a signal in the time or frequency domain. Here the uncertainty principle of the WOL is obtained.

First, two important equalities are provided

$$
\begin{aligned}
\int_{\mathfrak{R}} t^{n} W O L_{f}(t, u) d u & =\int_{\mathfrak{R}^{2}} t^{n} R_{f}(t, \tau) e^{\frac{i a}{b} \pi t} e^{\frac{i}{b} \tau\left(u_{0}-u\right)} d \tau d \tau d u \\
& =2 \pi|b| \int_{\mathfrak{R}^{2}} t^{n}|f(t)|^{2} d t
\end{aligned}
$$

And

$$
\begin{aligned}
\int_{\mathfrak{R}} u^{n} W O L_{f}(t, u) d t & =\int_{\mathfrak{R}^{2}} u^{n} R_{f}(t, \tau) e^{\frac{i a}{b} \pi t} e^{\frac{i}{b} \tau\left(u_{0}-u\right)} d \tau d t \\
& =2 \pi|b| \int_{\mathfrak{R}} u^{n}|\hat{f}(u)|^{2} d u .
\end{aligned}
$$

Let $n=2$, then the uncertainty principle of the WOL is obtained as below

$$
\begin{aligned}
& 2 \pi|b| \int_{\mathfrak{R}} t^{2}|f(t)|^{2} d t+2 \pi|b| \int_{\mathfrak{R}} u^{2}|\hat{f}(u)|^{2} d u \\
& =2 \pi|b|\left[\int_{\mathfrak{R}} t^{2}|f(t)|^{2} d t+\int_{\mathfrak{R}} u^{2}|\hat{f}(u)|^{2} d u\right] \geq \frac{\pi|b|}{2} .
\end{aligned}
$$

This shows that $W O L_{f}(t, u)$ cannot be sharply localized.

\section{CONCLUSION}

Based on the OLCT and the classical WVD theory, this paper proposes a novel kind of definition of WVD in the OLCT domain, namely WOL. Various properties of the WOL, including translation, modulation and multiplied signal are derived in details. Furthermore, a new approach to calculate the instantaneous frequency is presented. In addition, the uncertainty principle of the WOL is shown. The applications of the WOL in the nonstationary signal processing will be studied in our future papers.

\section{REFERENCES}

[1] S. C. Pei and J. J. Ding, "Eigenfunctions of the offset Fourier, fractional Fourier, and linear canonical transforms," Journal of the Optical Society of America A, vol. 20, no. 3, pp. 522-532, 2003.

[2] S. C. Pei and J. J. Ding, "Eigenfunctions of Fourier and fractional Fourier transforms with complex offsets and parameters," IEEE Trans. Circuits Syst. I, vol. 54, no. 7, pp. 1599-1611, 2007.

[3] Q. Xiang and K. Y. Qin, "Convolution, correlation, and sampling theorems for the offset linear canonical transform,” Signal Image Video Process., vol. 2014, no. 8, pp. 433-442, 2012.

[4] D. Urynbassarova, B. Z. Li, and R. Tao, "The Wigner-Ville distribution in the linear canonical transform domain,” IAENG International Journal of Applied Mathematics, vol. 46, no. 4, pp. 559-563, 2016.

[5] S. Abe and J. T. Sheridan, "Optical operations on wave functions as the Abelian Subgroups of the special affine Fourier transformation,” Opt.Lett. vol. 19, no. 22, pp. 1801-1803, 1994.

[6] R. Bracewell, The Fourier transform and its applications, 3rd ed., New York: McGraw-Hill Book Co., 1986.

[7] H. M. Ozaktas, M. A. Kutay, and Z. Zalevsky, The fractional Fourier transform with applications in optics and signal processing. New York: Wiley, 2000.

[8] T. Z. Xu and B. Z. Li, Linear canonical transform and its application. Beijing: Science Press, 2013.

[9] S. C. Pei and J. J. Ding, "Relations between fractional operations and time-frequency distributions, and their applications," IEEE Transactions on Signal Processing, vol. 49, no. 8, pp. 1638-1655, August 2001.

[10] R. F. Bai, B. Z. Li, and Q. Y. Cheng, "Wigner-Ville distribution associated with the linear canonical transform," Journal of Applied Mathematics, vol. 2012, pp. 1-14, 2012.

[11] M. Bahri and R. Ashino, "Convolution and correlation theorems for Wigner-Ville distribution associated with linear canonical transform,” 12th International Conf. on IT, 2015.

[12] Y. E. Song, X. Y. Zhang, C. H. Shang, H. X. Bu, and X. Y. Wang, "The Wigner-Ville distribution based on the linear canonical transform and its applications for QFM signal parameters estimation,” Journal of Applied Mathematics, vol. 2014, 8 pages, 2014.

[13] Z. C. Zhang, "New Wigner distribution and ambiguity function based on the generalized translation in the linear canonical transform domain," Signal Processing, vol. 118, pp. 51-61, January 2016.

[14] Z. C. Zhang, "Novel Wigner distribution and ambiguity function associated with the linear canonical transform," Optik-International Journal for Light and Electron Optics, vol. 127, pp. 4995-5012, 2016.

[15] J. Zhao, R. Tao, Y. L. Li, and Y. Wang, "Uncertainty principles for linear canonical transform,” IEEE Transactions on Signal Processing, vol. 57, no. 7, pp. 2856-2858, 2009.

[16] Y. G. Li, B. Z. Li, and H. F. Sun, "Uncertainty principles for WignerVille distribution associated with the linear canonical transforms," Abstract and Applied Analysis, vol. 2014, pp. 1-9, May 20147

[17] J. W. Goodman, Introduction to Fourier optics, 2nd ed., New York: McGraw-Hill, 1988.

[18] X. G. Xia, "Discrete chirp-Fourier transform and its application to chirp rate estimation,” IEEE Transactions on Signal Processing, vol. 48, no. 11, pp. 3122-3133, 2000.

[19] M. Wang, A. K. Chan, and C. K. Chui, "Linear frequency-modulated signal detection using Radon-ambiguity transform,” IEEE Transactions on Signal Processing, vol. 46, no. 3, pp. 571-586, 1998.

[20] S. Barbarossa, "Analysis of multicomponent LFM signals by a combined Winger-Hough transform,” IEEE Transactions on Signal Processing, vol. 43, no. 6, pp. 1511-1515, 1995. 